\title{
La leçon au Collège de France
}

Notes sur la transmission orale des savoirs à partir de quelques avantdire de Valéry, Merleau-Ponty et Foucault

\section{Benedetta Zaccarello}

\section{(2) OpenEdition}

1 Journals

\section{Édition électronique}

URL : http://journals.openedition.org/genesis/1382

DOI : 10.4000/genesis.1382

ISSN : 2268-1590

Éditeur :

Presses universitaires de Paris Sorbonne (PUPS), Société internationale de génétique artistique littéraire et scientifique (SIGALES)

Édition imprimée

Date de publication : 17 novembre 2014

Pagination : 71-84

ISBN : 9782840509714

ISSN : 1167-5101

\section{Référence électronique}

Benedetta Zaccarello, « La leçon au Collège de France », Genesis [En ligne], 39 | 2014, mis en ligne le 15 décembre 2016, consulté le 01 mai 2019. URL : http://journals.openedition.org/genesis/1382 ; DOI : 10.4000/genesis.1382 


\title{
La leçon au Collège de France Notes sur la transmission orale des savoirs à partir de quelques avant-dire de Valéry, Merleau-Ponty et Foucault
}

\author{
Benedetta Zaccarello
}

$I^{\prime}$ y a, à l'origine des pages qui suivent, l'idée que le dispositif même de la profération d'un cours au Collège de France contraint l'orateur à un régime de parole réflexif et paradoxal. Réflexif, il est facile de comprendre pourquoi, puisque l'homme de savoir appelé à prononcer ce genre de leçon l'est en tant que personnalité reconnue : l'élection au Collège de France «sanctifie », au sens étymologique du terme, la figure d'un penseur ou d'un savant, lui reconnaît un rôle majeur dans la vie de la discipline qu'il incarne et lui demande de canoniser pour ainsi dire sa propre œuvre en tant qu'apport original au patrimoine intellectuel commun. Il en découle une parole au statut forcément ambigu, tiraillée entre la personnalisation du savoir et la nature générale, voire théorique, des contenus que le même orateur est convié à partager avec son public.

Face à l'auditoire virtuel du pays tout entier, face aussi aux confrères idéalement installés aux premiers rangs, l'orateur se trouve ainsi pris dans un jeu de miroirs complexe. Tout en incarnant sa discipline, il délivre une image de lui-même, qui souvent, étant donné que l'on est généralement élu tardivement, finit par être un dernier mot sur lui-même. Il est vrai que toute parole, comme tout écrit, construit une image de son producteur, mais la situation orale a ceci d'exceptionnel qu'elle montre simultanément l'œuvre et son faber, tandis que, comme le rappelle sans cesse Valéry, ces deux entités restent normalement bien distinctes pour le lecteur d'un texte imprimé. Voici donc que l'orateur en question, en tant que je mais aussi en tant que représentant premier d'une communauté scientifique, se trouve conduit à brosser (et à contempler) à la fois un portrait de lui-même en savant, et celui de la discipline dont il devient volens nolens le représentant.

\section{Intitulé de la chaire et positionnement disciplinaire}

De cette situation, les trois cas que nous allons étudier sont assez emblématiques. Au moment de définir le titre de la chaire qu'ils doivent occuper, ils vont adopter des stratégies différentes, mais qui témoignent toutes trois d'un même malaise. Ainsi, appelé en 1937 à enseigner la «Poétique », Valéry, qui se sait connu principalement comme auteur de vers, n'ose plus changer le titre retenu pour l'enseignement qui lui a été confié1. Il ne cesse toutefois de dire que ce qu'il se promet d'enseigner n'est pas ce qu'on entend couramment par le mot poétique et prend la liberté de redonner à celui-ci son sens étymologique. Ce qui l'intéresse, c'est ce qu'il appelle la «Poḯtique »: l'analyse des dynamiques propres à tout acte de création. Par là, il modifie le périmètre de la discipline qui lui est assignée et, sans intervenir dans l'intitulé, opère un décalage substantiel vers l'esthétique et la philosophie. De son côté, Merleau-Ponty non seulement garde pour sa chaire le sobre intitulé de «Philosophie », mais, comme le veut le titre de sa leçon inaugurale de 1951, il commence par faire l'éloge même de sa discipline ${ }^{2}$. Toutefois, il consacre sa première année au Collège à des domaines hybrides, touchant davantage la psychologie et la littérature. Par là, il entend implicitement redessiner la carte des territoires que la philosophie contemporaine doit désormais s'approprier. Quant à Michel Foucault, il n'hésita pas en 1970 à réintituler sa chaire de façon assez révélatrice.

1. Voir les remerciements de la leçon inaugurale dans Paul Valéry, «Première leçon du cours de poétique », Euvres, t. I, Paris, Gallimard, coll. « Bibliothèque de la Pléiade », 1957, p. 1340.

2. Publiée justement sous le titre «Éloge de la philosophie », dans Éloge de la philosophie et autres essais, Paris, Gallimard, 1953. 
Alors que Jean Hyppolite avait semblé rétrécir les ambitions de la discipline en transformant l'" Histoire et technologie des systèmes philosophiques » de Martial Gueroult en une «Histoire de la pensée philosophique », il se défait de tout « fétichisme » philosophique et opte pour « Histoire des systèmes de pensée ». S'il réintroduit la notion de « système » dans son intitulé, ce n'est plus pour l'associer au travail des concepts, mais, bien au contraire, pour en solliciter une analyse critique.

Or, la question des positionnements disciplinaires engage le statut de la vérité que sous-tend l'activité pédagogique. En d'autres termes, le régime de réflexivité questionne, outre la personnalité de l'orateur et les limites de sa discipline, la véridicité des paroles prononcées, dans une sorte de renversement du paradoxe du menteur : celui qui parle affirme implicitement qu'il dit « vrai », or, parce qu'il questionne son propre point de vue et son œuvre, mais aussi le langage théorique dans lequel il s'exprime, voire les limites dans lesquelles nous pouvons espérer tenir un discours vrai, le professeur se trouve à la fois incarner et contester ces mêmes éléments, endosser et critiquer l'image qu'il projette pour son auditoire.

À travers ces trois exemples, dans une lecture serrée de quelques « avant-dire ${ }^{3}$ », nous essaierons de penser le fructueux paradoxe qui semble surgir de l'asymétrie constitutive du régime de réflexivité spécifique des cours du Collège de France, situation où entrent en conflit la valeur symbolique de l'institution et le perspectivisme implicite de tout acte d'enseignement. Le jeu et le « théâtre » de la leçon au Collège de France, du moins au prisme des acteurs que nous avons choisis, permettent de voir que la question du temps est cruciale pour tracer une démarcation entre oralité et œuvre publiée, entre discours et littérature, entre acte de parole et choix de la publication. Car dans les humanités, si le professeur parle, c'est d'abord avec la légitimité des livres qu'il a écrits et au sujet d'autres textes eux aussi publiés et accessibles à son auditoire. Si le professeur parle, c'est bien à propos d'écrits, mais pour en évaluer ou déplacer la valeur de vérité par la vertu même de la parole vive.

\section{Imago sui : Paul Valéry ou la poïétique de soi-même}

Lorsque Valéry est élu au Collège de France, en 1937, c'est d'abord en tant que poète. Cependant, sa production en prose, sollicitée par d'innombrables obligations intellectuelles ou officielles, lui a permis de faire émerger une théorie qui, après avoir longuement mûri dans le kykeon des Cahiers, a été progressivement livrée au public par à-coups et selon les contraintes du moment. Cette prédilection pour la rédaction fragmentaire et pour l'inachevé ne doit toutefois pas faire oublier sa quête permanente d'une systématisation. Or, si, en 1937, Valéry a renoncé à ramener les notes des Cahiers dans le périmètre d'une doctrine ou d'une métadoctrine (aspiration qui fut davantage présente dans ses années de jeunesse, au moment où le projet même est conçu sous le signe du « Système »), il a dû également faire le deuil d'une publication substantielle de ses notes.

Voici pourtant que les circonstances semblent le ramener à son souhait premier. Quelle meilleure occasion en effet que les leçons du Collège pour réorganiser de manière systématique sa propre pensée ? C'est précisément dans cette direction que Valéry semble orienter son enseignement, comme il l'explique dès les premières lignes de sa leçon d'ouverture :

\begin{abstract}
Vous avez peut-être pensé que certaines matières qui ne sont pas proprement objet de science, et qui ne peuvent pas l'être, à cause de leur nature presque tout intérieure et de leur étroite dépendance des personnes mêmes qui s'y intéressent, pouvaient cependant, sinon être enseignées, du moins, être en quelque manière communiquées comme le fruit d'une expérience individuelle, longue déjà de toute une vie, et que, par conséquence, l'âge était une sorte de condition qui, dans ce cas assez particulier, se pouvait justifier ${ }^{4}$.
\end{abstract}

Appelé à prendre la parole en tant que poète, Valéry n'entend pas s'en tenir au seul champ de la poésie proprement dite. Il transforme d'emblée son cours en laboratoire d'une théorie du faire, qui exige une méthodologie assez différente de ce que les études littéraires de l'époque pouvaient proposer :

3. Les documents utilisés ont des statuts différents : Valéry a publié luimême, et à plusieurs reprises, sa leçon inaugurale sur la poétique ; nous disposons de notes prises par Merleau-Ponty pour servir de support à son enseignement ; et les cours de Foucault ont été enregistrés et sont cités ici à partir de leur transcription.

4. P. Valéry, « Première leçon du cours de poétique », op. cit., p. 1342. 
J'ai donc cru pouvoir le reprendre [le mot Poétique] dans un sens qui regarde à l'étymologie, sans oser cependant le prononcer Poïétique [...]. Mais c'est enfin la notion toute simple de faire que je voulais exprimer. Le faire, le poïein, dont je veux m'occuper, est celui qui s'achève en quelque œuvre et que je viendrai à restreindre bientôt à ce genre d'œuvres qu'on est convenu d'appeler ceuvres de l'esprit. Ce sont celles que l'esprit veut se faire pour son propre usage, en employant à cette fin tous les moyens physiques qui lui peuvent servir 5 .

Dans cette démarche, la position de Valéry se dédouble : l'orateur est aussi l'objet de sa propre théorie, puisque son expérience de poète doit lui servir pour fonder une étude analytique des dynamiques propres à tout geste de création (fig. 1).

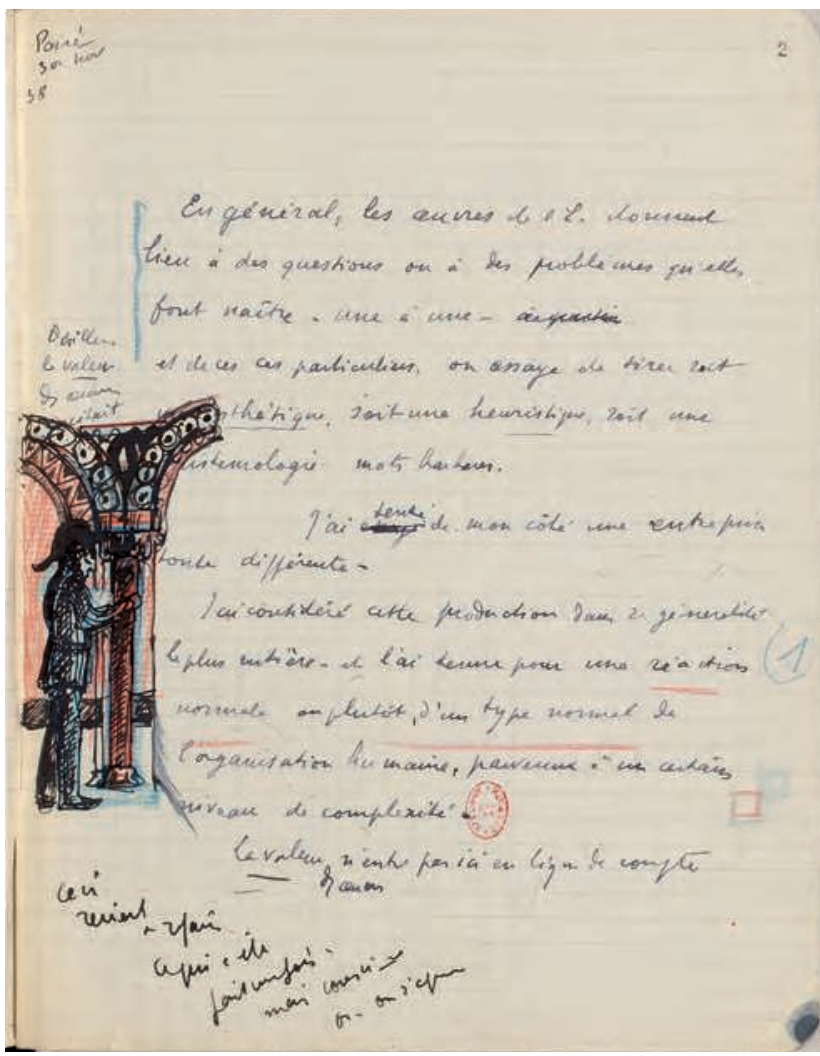

Fig. 1 : BnF, fonds Paul Valéry, NAF 19099, fo 2
Parallèlement au séminaire où il lit avec ses disciples des textes qui lui semblent illustrer les résultats auxquels est parvenu son enseignement magistral, Valéry esquisse, au cours de ses premières années au Collège, un autoportrait théorique, en « créateur quelconque », brossé selon les traits «neutres » de l'auteur en tant que tel. Or, bien que cet élément de réflexivité soit mis en avant dès la leçon inaugurale et justifié par des considérations de nature épistémologique, cela n'est pas sans entraîner des difficultés. En effet, comment la Poétique peut-elle prétendre être un savoir digne d'enseignement, alors qu'elle traite des ombres fuyantes de la conscience de l'auteur ? Comment fonder la légitimité de ce qui est dit pendant les cours, alors que l'objet de ces derniers est, par définition et selon la théorie esthétique de Valéry lui-même, inaccessible au lecteur? L'auditeur pourrait-il donc, grâce à l'épiphanie de l'auteur lui-même en chair et en os, avoir accès à ce qui précisément, selon Valéry, lui est refusé en tant que lecteur?

De cette difficulté témoignent les cahiers utilisés par Valéry pendant la préparation de son cours. La question des conditions nécessaires pour qu'un savoir puisse être considéré comme scientifique apparaît ainsi au sein de ces notes éparses. Elle y est précisément posée à partir de ces éléments : l'impersonnalité du savoir, la capacité qu'ont les énoncés à être indépendants des sujets, l'extériorité observable des phénomènes décrits :

Et même, il n'y a science que lorsque / cette séparation [entre l'objet et les méthodes] semble entièrement réussie.

Lorsque les résultats semblent tout indépendants des personnes - et non seulement des personnalités / mais des [illis.] facultés 6 .

Parallèlement, l'idée valéryenne de l'auteur comme « mythe » produit par le lecteur est ici non seulement reprise mais radicalisée :

Il y a une confusion dans le vague où ces questions sont généralement retenues par l'usage [...] entre l'auteur de

5. Ibid.

6. Bibliothèque nationale de France, fonds Valéry, NAF 19096, fo 7 ro. 
l'œuvre - et ce qui fait l'œuvre. L'auteur est un mythe visible, un individu relativement impénétrable. Mais on a beau le connaître cette connaissance est extérieure, et de cette connaissance l'œuvre ne peut résulter comme l'opération de cet individu. Nous savons seulement que l'œuvre est le produit final d'une modification de cet être Modification qui intéresse son milieu - qui se dessine, se développe et se résout, s'achève, replongeant le système vivant dans son état initial - cependant qu'il se détache de lui et prend place dans le milieu un effet sensible extérieur7.

La chose paraissait d'abord simple : un poète était appelé à parler de son art. Mais voici que la chose se complique dans un jeu de miroirs et de mises en abyme. L'orateur en effet ne peut parler de son geste créateur sans faire référence à la fois à sa propre expérience et aux produits de son activité créatrice. Cela ramène Valéry à la tension dialectique qui lui est chère depuis toujours entre la personnalité et l'œuvre, entre l'auteur et cet objet mystérieux qui, issu d'un vécu si intime qu'il ne pourra jamais être partagé, n'appartient pas plus à son créateur qu'à un quelconque lecteur. Mais dans les notes préparatoires du cours de 1937-1938, l'idée de l'extériorité de l'œuvre à son auteur semble tout particulièrement accentuée. Tout comme le corps s'oppose à l'esprit, l'œuvre s'opposerait d'une part au monde, de l'autre à l'esprit lui-même :

Nous et notre esprit $\quad \mathrm{CEM}^{8}$

Idée curieuse que nous en avons.

Opposition - et confusion, entre moi et mon esprit.

Variabilité / Il y a donc une distinction, un intervalle / aussi marqué que celui avec notre corps - / à ce point que le possessif - mon - est relatif

Lutte contre les parasites.

\section{L'incertitude}

$[\ldots]$

L'œuvre (de l'esprit) / s'oppose à l'esprit - / L'esprit luimême s'oppose / à... MOI.

au Monde et / au Corps.

Moi toujours tend à être hors du jeu 9

Tout en insistant sur la séparation de l'œuvre et de l'auteur, Valéry accentue la dimension performative implicitement contenue dans la notion de poïétique. Puisqu'il ne peut y avoir de science que de ce qui est externe au sujet impliqué et de ce qui a des retombées observables, Valéry va développer une théorie du faire qui prend appui sur l'aspect performatif de son esthétique. L'ontologie qui en résulte voit en l'œuvre moins la somme des états successifs de sa production, ce à quoi Valéry nous a davantage habitués, que l'action même qui en est à l'origine :

Le sensible et le faisable sont les moyens nécessaires - de la transmissibilité

En somme.

la notion d'œuvre est une notion d'action / (considérée du côté de sa production - )

Cette action exige une modification particulière / une mise en train, une coordination, un / groupement non permanent dans l'individu-auteur / et nous devons - avant toute chose, essayer d'isoler / ce système - de l'imaginer - et de noter / aussi tous les obstacles que nous trouvons à $\mathrm{n}[\mathrm{ou}] \mathrm{s}$ faire / cette représentation $10[\ldots]$

Nous avons besoin, avant toute chose d'une / sorte de formule d'action - qui nous permette de / suivre un genre d'action si complexe - - sans trop

$\mathrm{n}[\mathrm{ou}] \mathrm{s}$ écarter de ce que nous considérons comme l'ordre / naturel des phénomènes $-[\ldots]$

Il est évident que cette formule / d'action doit être aussi générale que possible / générale signifiant d'abord que les actions les plus variées puissent être rapportées à elle [... $]^{11}$ (fig.2).

Si les modifications subies par celui qui crée sont des mystères insondables, on peut en tout cas les appréhender en termes d'actions et définir ce qui se passe en amont de l'œuvre en tant que « travail ». C'est pourquoi, semble-t-il,

\section{Ibid., fo 3 ro.}

8. Ce sigle, dont les Cahiers font un large usage, indique chez Valéry le plexus constitué par Corps Esprit et Monde. Ces trois polarités sont observées par Valéry à la fois dans leur interrelation profonde et dans leur distance irréductible, l'acronyme lui servant justement pour mettre en évidence le champ tracé par cette même relation et coïncidant finalement avec le périmètre d'action possible de la conscience.

9. Ibid., fo 1 ro.

10. Ce passage est souligné par un trait vertical en marge, précédé par l'indication d'un « $\mathrm{P} »$ majuscule.

11. Ibid., fo 4 ro. L'expression « sans trop nous écarter de l'ordre naturel des phénomènes » semble suggérer que Valéry cherche à faire une sorte de «physique » de l'acte créatif. 


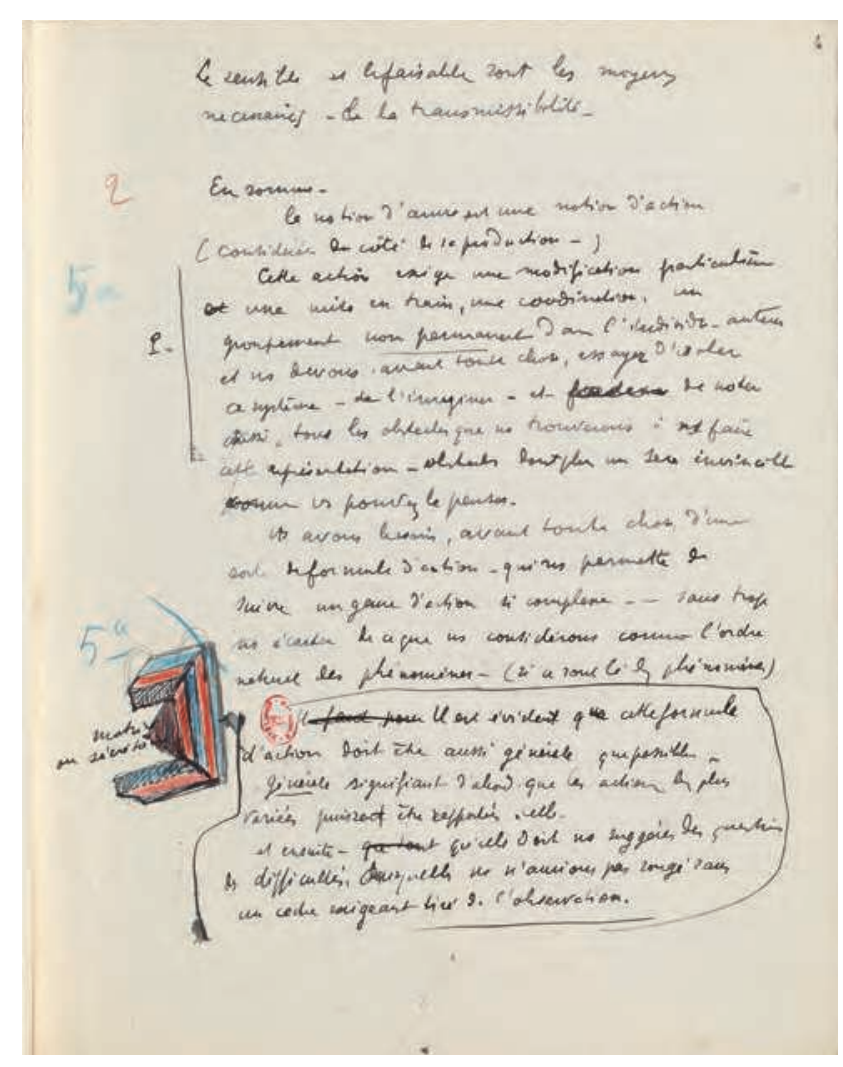

Fig. 2 : BnF, fonds Paul Valéry, NAF 19096, fo 4

Valéry, qui avait précisé dès la leçon inaugurale ne vouloir s'intéresser qu'aux œuvres de l'esprit envisagées dans les dynamiques de leur «production », est amené dans ces notes à introduire la notion de «travail mental 12 », ce qui semble encore une fois problématique pour sa propre esthétique. Est ici baptisé « Action complète » (fig. 3) l'aboutissement de ce travail mental, le paradigme de son achèvement et de sa réussite, là où la complétude de cette action supposerait justement un élément extérieur et non personnel :

Pas de vue sans tangibles

Pas de bruit sans émetteurs [...]

Pas d'intervalle sans liaison-distance

Pas d'événements distincts sans remplissage

On peut donc supposer [...] que nous / possédons, à titre fonctionnel constant, un / type, une forme qui exige ces formations
Cette forme est celle de l'Action complète

- qui est donc le maximum de compréhension

- qui fournit les lacunes, et tend à / engendrer les réponses naïves (fig. 4).

[...]

L'Action Complète demande un élément, ou ingrédient non-moi13 (fig. 3).

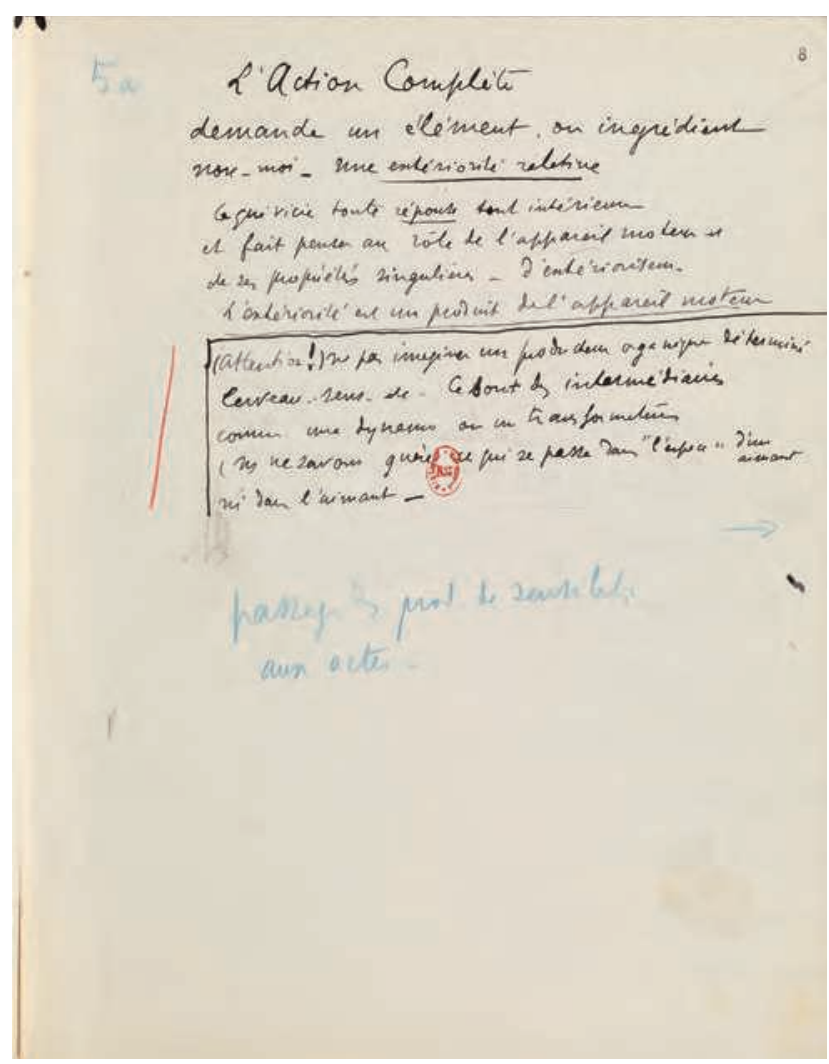

Fig. 3 : BnF, fonds Paul Valéry, NAF 19096, fo 8 ro

Il serait alors parfaitement légitime de produire une science du faire et du faire poétique. Une telle interprétation de la stratégie argumentative de Valéry, vouée implicitement à justifier en quelque sorte la légitimité même de son propos au moment précis de sa profération, permettrait aussi de comprendre la

12. NAF 19096, fo 1 ro.

13. Ibid., fo 7 vo et 8 ro. 


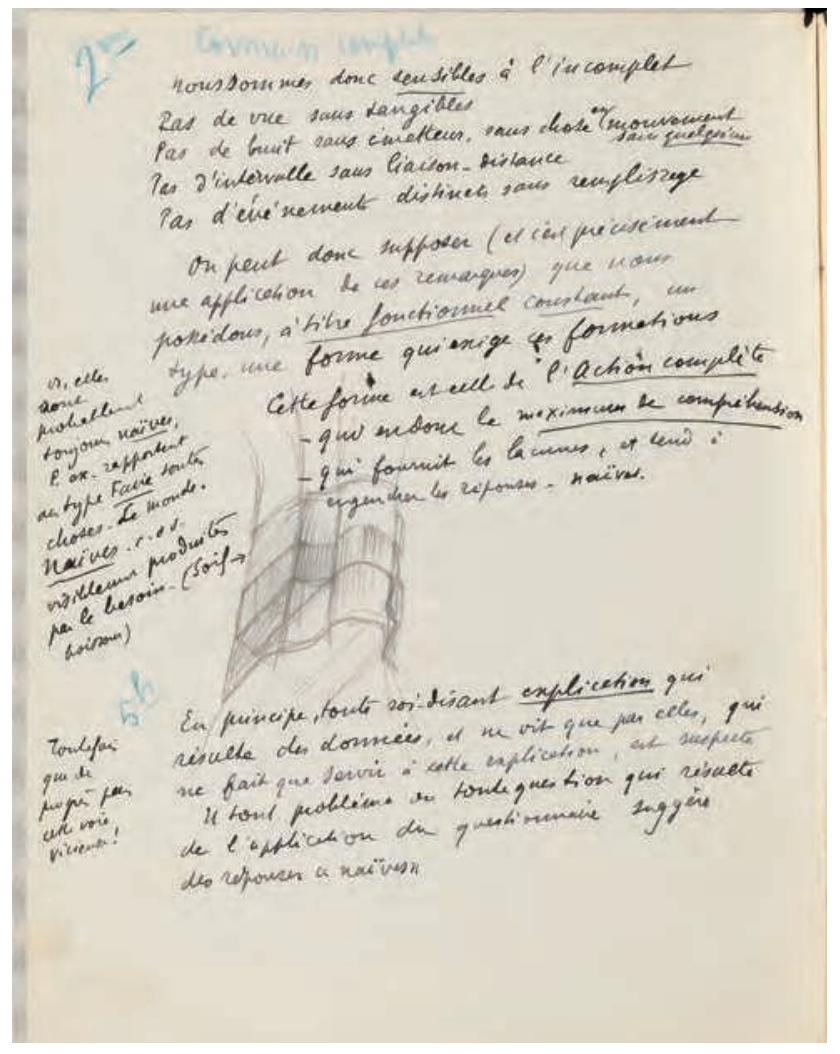

Fig. 4 : BnF, fonds Paul Valéry, NAF 19096, fo 7 vo

radicalisation de l'opposition entre auteur et œuvre que Valéry envisage dans ces mêmes ébauches de leçon, où l'auteur est décrit comme totalement dépossédé de sa création, comme pur récepteur ${ }^{14}$. Tout comme, selon la physique quantique, l'œil de l'observateur brouille les données et les phénomènes, l'esthétique valéryenne se trouve à la fois troublée et radicalisée. Il est en ce sens intéressant que, dans ses brouillons successifs, Valéry semble prendre ses distances avec les analyses développées auparavant, non parce que celles-ci seraient fausses, mais parce qu'elles seraient difficiles à comprendre pour son auditoire. De plus, en marge de notes concernant le contenu théorique de ses cours, il commence à rédiger des parties dont le registre est celui de l'oralité propre à ses leçons, comme si la parole pouvait en quelque sorte venir achever l'œuvre, voire en livrer une plus solide vérité.
Ce mouvement est particulièrement sensible dans les avant-dire des premiers cours de la deuxième année, que Valéry semble noter tels qu'il voudrait les prononcer : le ton et le registre diffèrent de ses derniers essais esthétiques et font entendre la voix qui va les proférer. C'est à ce stade du cours qu'il revient sur ses principes méthodologiques et semble presque s'excuser de la hardiesse de spéculations dont il connaît à la fois l'originalité et la densité théorique.

Si je me suis progressivement avaneé dans < fait $>$ cette manière de voir dont les considérations que je v[ou]s expose dans ce cours sont, en somme, l'analyse (aussi précise que je puis le faire) - c'est eomme en que telle autre manière de penser aux choses de l'esprit ne s'adaptait pas au mien - Tel langage - tel vocabulaire ne touchait pas en moi le point où le fonctionnement $<$ variable $>$ de la vie, la représentation des choses, les valeurs [...] sont simultanément intériorisées (fig. 5 et 6 ).

C'est donc la pratique même du cours qui révèle progressivement à Valéry à la fois le perspectivisme implicite à sa théorie, sa différence par rapport aux langages traditionnellement utilisés pour décrire ce genre de phénomènes, et la difficulté de traduire la sensibilité propre à un esprit, dans un vocabulaire abstrait qui puisse être compris et partagé par la communauté idéale de ses auditeurs 15 . En cela, les leçons de Poétique témoignent à la fois du «travail mental » dans lequel consiste toute œuvre et de l'effort de compréhension que cette dernière exige, à son auteur comme à tout public. Et c'est cette double difficulté, du professeur et de son auditoire, qui explique probablement le désir de complicité que trahit l'allure discursive de cette rédaction.

\section{Langages indirects. Maurice Merleau-Ponty et l'anti-philosophie}

Bien que, d'après les notes dont nous disposons, Maurice Merleau-Ponty n'ait pas thématisé dans ses leçons au

14. Outre l'élément « non-Moi » que nous venons d'analyser, le folio 10 ro suggère que «L'individu se fait instrument » quand il s'agit de production esthétique. L'œuvre de l'esprit serait telle que « qui la subit, la “crée”, se sent la créer » (fo $\left.1 \mathrm{v}^{\mathrm{o}}\right)$.

15. Comme l'attestent à plusieurs reprises les pages suivantes de ce cahier où Valéry fait allusion à la difficulté de ce cours. 

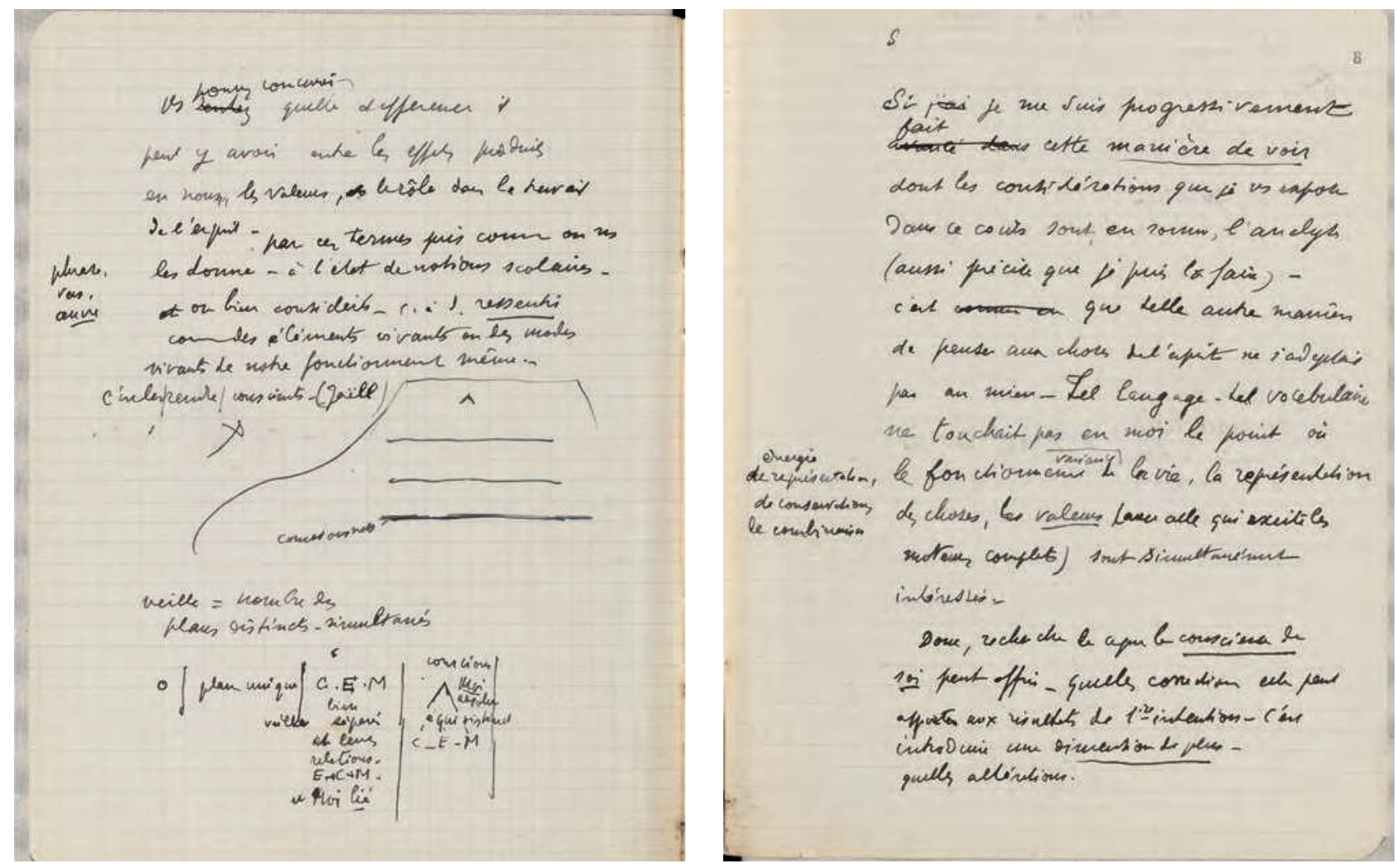

Fig. 5 et $6:$ BnF, fonds Paul Valéry, NAF 19099, fos 7 vo et 8 ro

Collège de France la spécificité de sa position professorale, il a développé, dès le début de son enseignement, une réflexion sur l'écrit et l'oral qui n'est sans doute pas étrangère à la nature de la prestation elle-même et engage, à ce titre, une forme de réflexivité. C'est du moins une des lectures que l'on peut donner de la redéfinition de la littérature contemporaine comme régime de parole dans les avant-dire des «Recherches sur l'usage littéraire du langage », un des deux cours proposés par Maurice Merleau-Ponty lors de sa première année dans l'institution.

Les lignes qui suivent sont empruntées aux notes de la deuxième séance de cet enseignement ; elles appartiennent encore à la partie introductive :

Prose et poésie - Rapport synthétique à autrui Imaginaire et vérité : la vie ne se comprend qu'à la 1 re personne, dans <monologue créateur $>$ ce qu'on a à dire et la technique
Écrire et sincérité [...]

Dans quelle mesure écrire est-il penser son œuvre, dans quelle mesure elle échappe et l'écrivain fait autre chose que ce qu'il voulait

Anti-littérature dans la littérature

[...]

Écrire et vivre : écrire justifie-t-il, dispense-t-il de vivre? ou bien vivre d'écrire?

Tout cela tourne autour du problème : le vrai

autrui
soi
dans la littérature
dès qu'on ne croit plus au vrai
ou universel où l'on s'installe16

(fig. 7).

16. M. Merleau-Ponty, Recherches sur l'usage littéraire du langage, éd. B. Zaccarello et E. de Saint Aubert, Genève, MétisPresses, 2013, p. 85-86. 


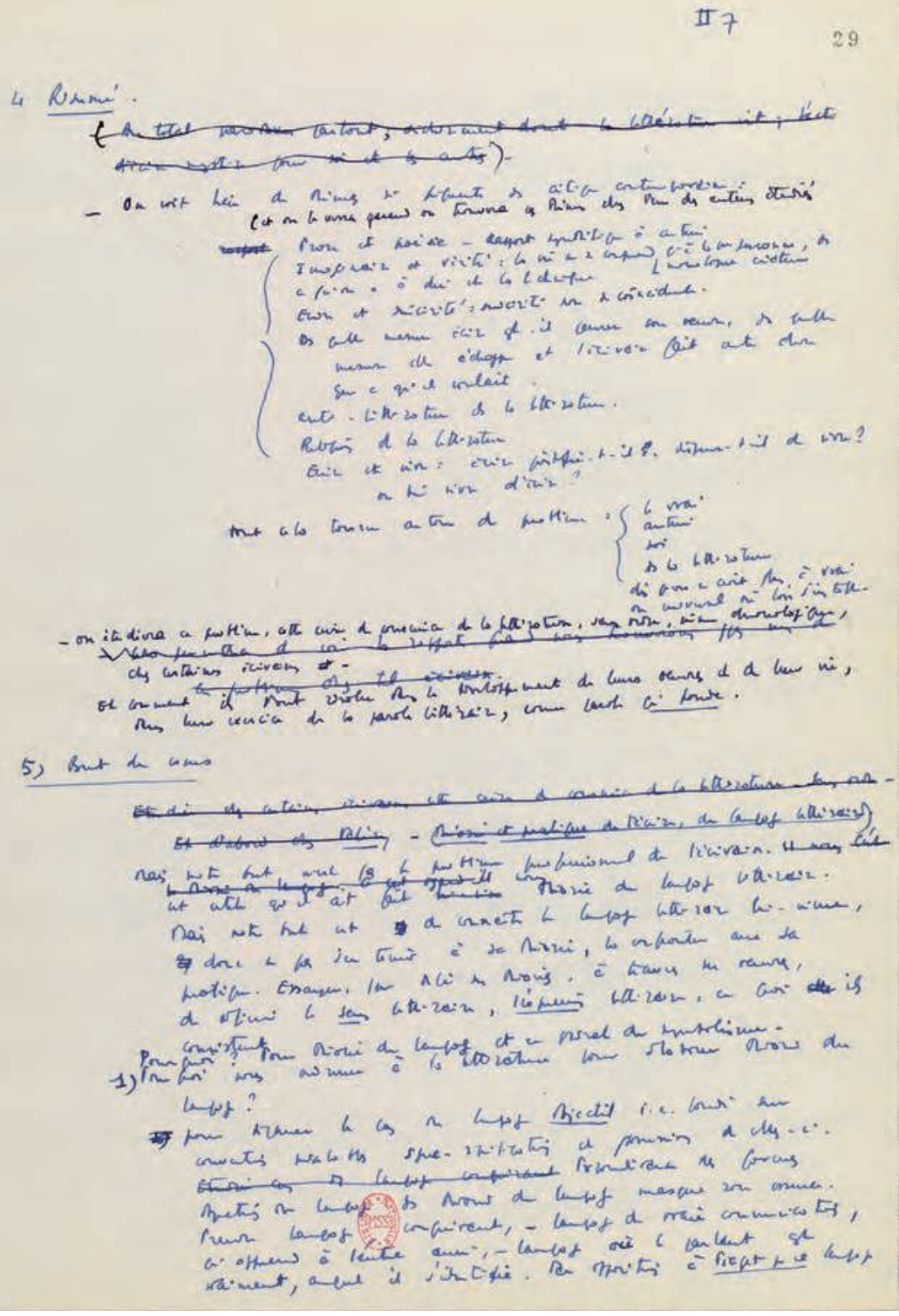

Fig. 7 : Maurice Merleau-Ponty, notes pour le cours « Recherches sur l'usage littéraire du langage » (BnF, fonds Merleau-Ponty, NAF 26994 MF 9846, fo 29 ro)

La littérature se configure donc ici comme « rapport synthétique à autrui ». Synthétique parce qu'elle doit combiner l'intention muette qui anime le sujet parlant/ écrivant et l'apport créatif fourni par celui qui reçoit ce message. Après avoir constaté la survivance de la philosophie malgré la révélation, à la fin de la guerre, de son incapacité à comprendre le monde, on doit accepter que rien ne se laisse complètement figer dans un tissage conceptuel : le «réel » trahit constamment l'image que le langage prétend en donner. Un homme qui parle est un homme qui «trahit » sa pensée ; un homme qui écoute est un homme qui trahit la parole d'autrui. Toute parole sur la vie s'avère alors un objet paradoxal puisque, ne pouvant être « sincère »qu'en tant que monologue intérieur, elle ne se donne pourtant que comme dialogue implicite avec un lecteur. Ce rôle déterminant du lecteur avait déjà été révélé par Valéry et Stendhal autour de qui, justement, s'articule le cours. Ce n'est pas un hasard si, dans les portraits qu'en donne Merleau-Ponty, ces deux écrivains ne parviennent à l'écriture qu'après une expérience du silence et avec un scepticisme radical face au langage et face à leur propre capacité à exprimer quelque chose de « sincère ».

Ce régime particulier du rapport synthétique à autrui dans la littérature contemporaine, Merleau-Ponty le nomme « anti-littérature » :

«Si l'auteur se connaît un peu trop, si le lecteur se fait actif, que devient le plaisir, que devient la littérature ? »(Variété III p. 65)17. Et c'est cependant cette anti-littérature qui est pour lui la Littérature 18 .

La littérature apparaît bien ici condamnée à se transformer immanquablement en anti-littérature : elle se donne uniquement dans sa valeur performative, dans le geste de l'écriture ou dans celui de la lecture, au moment où l'écrit se montre en tant que profération, éternel retour de l'hic et nunc, dans un changement perpétuel sur lequel échoue toute volonté de fixer ce qui, dans l'expérience, se donne comme jaillissement momentané :

La poésie est voix, faire, non dire

Elle n'est pas voix au sens de l'homme avant qu'il sût écrire (que serait la poésie de Valéry sans l'écriture ?) - [...] $<$ Or la voix,

en ce sens là, existe [...] aussi dans la prose> (fig. 8) ${ }^{19}$.

17. P. Valéry, Euvres, t. I, op. cit., p. 1500-1501.

18. M. Merleau-Ponty, Recherches sur l'usage littéraire du langage, op. cit., p. 108.

19. Ibid., p. 137. 


$$
\text { Langue et litterature vi Is }
$$

and bus to more

toot, ex dis celle de utenansi selon V.: "on entend quel puerois Eclater dans le texte, comine des theises tout powonnels, celiaines locutions, pres ue wos interjections, yil n'ont u'une valeur de aicntux nervelax... ecaiae $i$, su ailieu de circonstances médioores ou assomantes, contre l'exces a'euni ou de nelancolie, contre 1

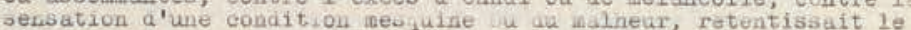
timbe tout pulasent de 1. valeur personnelle, le cri a' ulerte de 1.unique soi-mene.

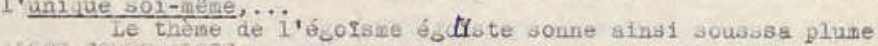
ALCHS COSL ALORS cutre thème, celui des nilets $t$ ops hauts" ( $\operatorname{var} 11$ p y1)

c'est cette voix-1a, (qu'on peut entenare en prose, ce qui ne veut pas dire qu'elle est prosaique: elle eat 1a partie aetive, non-infor

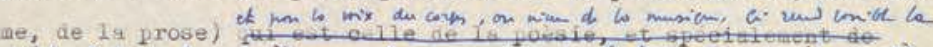

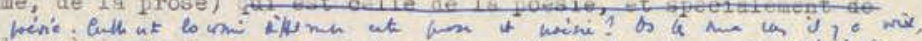

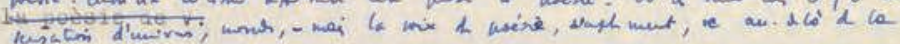
lunoune L'orelile un pontile bilare S'aventare vers le iutur: vae attente sainte la pencue Car une volx nouvelie et bianone tenappe de ce corps impur:

Homneur des Eomides, saint hangate

Discours propheti le et paró

Delles enâlines en qui s'engaje

Le cieu dans la chafr egaré

voici parler we stuease

t sonitr cette auche voix

Hi sonitr cette aususte voli

Lit se connait quand elle sonne

rant que des ondes et des bois!

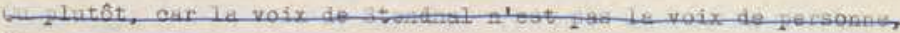

La voix de joèsie est une voix des rinoses, Ia prononciation de

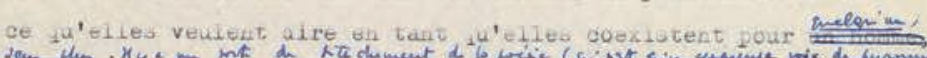

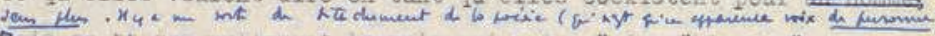

Bais en t. cis wuilewent la vols jui grie. Voix, "blanone", par op-

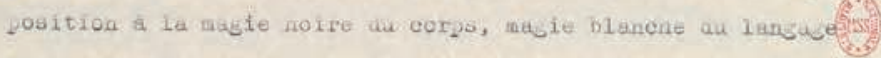

$i=$ sa cuatilité, son poavoir a'articuler et de lazre voir par des

(x)

Fig. 8 : Maurice Merleau-Ponty, notes pour le cours "Recherches sur l'usage littéraire du langage » (BnF, fonds Merleau-Ponty, NAF 26994 MF 9846, fo 75 ro)

En cela, l'anti-littérature semble se configurer davantage comme un régime de parole que d'écriture. Car, pour être "sincère », c'est-à-dire pour se connaître en tant que vaine tentative de sincérité, elle doit constamment répondre à l'instabilité de la vie, au mouvant de l'expérience. Pas de littérature sans exercice de vie, cette idée s'incarnant parfaitement dans la figure de Stendhal :

Stendhal écrivain et amateur ailleurs. Non homme de lettres ce qui suppose égard à autrui donné - Mais voix, parole, animation - « Il faut secouer la vie autrement elle nous ronge $\gg 20$.
Cette image stendhalienne de la vie « rongeuse » sera évoquée encore une fois dans les pages des Recherches sur l'usage littéraire du langage :

Littérature $=$ au plus haut point « $\underline{\text { secouer la vie }}$ » (« au-

trement elle nous ronge ») : passer outre à la difficulté d'être par

la dextérité de la parole, mouvement en avant qui nous donne à

autrui et lui à nous21.

Il ne s'agit pas, en somme, de vivre et de se regarder vivre, pour fabriquer ensuite de soi une image littéraire à l'usage d'un lecteur figé, « donné », mais de parvenir à une écriture qui puisse être elle-même «parole » et " animation », déjà incarnée, déjà mouvante ${ }^{22}$, comme l'était, en amont, l'expérience qui l'a portée, comme le sera, en aval, celle du lecteur «actif» :

Montrer dans le monologue intérieur la vraie parole, qui incorpore le silence, et qui ne suppose pas la sincérité par rapport à un moi donné, mais ni échec devant l'instantané, ni vanité d'être écouté par autrui, de se faire exister devant lui, tel qu'on se veut. Stendhal est l'apprentissage de tout cela : c'est pourquoi il sera un homme qui est écrivain et non un homme de lettres ${ }^{23}$.

20. M. Merleau-Ponty, Recherches sur l'usage littéraire du langage, op. cit., p. 194.

21. Ibid., p. 215

22. L'expérience vivante de l'auteur sera doublée par celle de la totalité des récepteurs, communauté qui traverse le temps. «Cependant 1) Les autres veulent parler aussi, z) łeurolut / s'intéressent à la littérature, qui commence avec la / parole Z). Leur vie se nourrit de vérité. La littérature n'est que / la récupération dans l'imaginaire de ce que la vie des choses / mêmes dont la vie est faite. Le problème est donc non celui / de l'écrivain seulement mais celui de l'homme » (ibid., p. 23). Dans les pages de ce cours, Stendhal est l'emblème de l'écrivain qui écrit pour la postérité, qui «[t]ravaille toujours pour le $\mathrm{XX}^{\mathrm{e}}$ siècle » et qui, par là même, accepte d'emblée qu'à ses paroles on donnera le sens qu'on voudra. Une fois de plus, on constate que dans l'analyse de Merleau-Ponty la littérature est un objet paradoxal, qui tend vers un régime performatif, quasi oral, mais ne peut être « entendue » que par la postérité.

23. Ibid., p. 197. 
Plusieurs éléments permettent ici de mieux comprendre ce que peut être cette anti-littérature que le monologue intérieur devrait incarner : elle relève le défi de l'instantané et ne se laisse pas figer dans un universel qui la trahirait ; elle n'est pas volonté d'être, mais performativité de l'être et apprentissage constant de cette même performativité ; elle n'est pas le territoire des hommes de lettres, mais des écrivains en action. Or, cette action est d'autant plus effective qu'elle est, une fois de plus, indirecte, ce que Merleau-Ponty exprime en la nommant « anti-action », utilisant un calque de l'expression même d'anti-littérature24. Mais surtout, la « parole » de la littérature, contrairement à celle du langage ordinaire, est capable d'incorporer le « silence » : seule la parole capable d'incorporer le silence réussit à s'approprier la vie 25 et est en ce sens le modèle et le paradigme toujours à venir de la littérature, le stade où celle-ci parvient à se dégager d'un universel creux et périmé (fig. 9).

Or, une parole ainsi prise dans sa valeur performative, constamment projetée vers l'avenir de ce qu'elle engendrera grâce à son enracinement dans l'instant présent, ressemble bien davantage à une parole prononcée qu'à un discours écrit :

Sartre dirait que parler c'est avoir con-/science de parler. Mais il faut dire aussi l'inverse : avoir conscience de parler, c'est parler / d'abord. Il n'y a pas de hylé sensible sans signification, mais dans la parole vivante, dans la parole-praxis, il n'y a pas non plus de mots sans une signification autochtone $[\ldots]^{26}$.

Dans ce nouveau régime parlé, la littérature ne correspond plus à une réalisation livresque, mais à une réalisation où, dans l'instant, le sens et son énonciation viennent à coïncider comme dans le langage oral. Dans l'oralité quotidienne, certes, la parole ne profère que des sens toujours donnés au préalable et qui, existant déjà en amont de l'acte d'énonciation, lui interdisent toute possibilité d'être en même temps un acte de pensée et une véritable profération. À l'inverse, l'anti-littérature, monologue toujours en train d'être prononcé pour un écouteur toujours à venir, est une mise au point constante de ce que les mots peuvent vouloir dire, dans de nouveaux potentiels sémantiques, dans les espaces silencieux et ouverts qui les relient :
Le langage et le sens ne font qu'un parce que c'est seulement en / « mettant au point » la machine à parler sur l'intention muette (comme en / mettant au point les yeux sur les images) qu'on en vient à penser ce qu'on / pense. Opération de l'être parlant qui n'est pas plus fondée sur possession / intellectuelle du langage propre que la fixation sur connaissance de l'optique / géométrique et de l'anatomie. Qui donc suppose polarisation de la parole / par ce qu'il y a à dire plus efficace que notre volonté délibérée de dire ceci / ou cela. Nous voulons dire ceci ou cela et les paroles que nous appelons / veulent dire autre chose qui devient l'œuvre. Un fonctionnement comme / naturel, un rapport d'échange s'établit entre l'intention muette et ce que / nous disons, l'appareil du langage est guidé par un sens que nous n'avons pas / intellectuellement, la « voix qui est » appelle la « voix qui vient» (Introduction / à la Poétique p. 41) et « qui doit venir», sans que nous sachions exactement pourquoi ${ }^{27}$.

Une littérature, en somme, à l'image de la philosophie dont Merleau-Ponty semble faire indirectement l'éloge dans ce même cours, philosophie émancipée de toute nostalgie des universels, seulement indirectement engagée, et s'exprimant non seulement dans les livres, mais aussi dans l'oralité d'un enseignement, celui justement de la leçon qui est en train d'être prononcée et qui semble être perçue non seulement en tant que prise de parole, mais aussi en tant que prise de position.

[- Rapport de la littérature et $<$ du sens $>$ de la vérité. [...]

Il faudrait montrer [...] comment il n'y a pas d'explication

24. « [...] la littérature, anti-action, / est cependant, et à ce titre même, toute prise dans l'action, susceptible d'une / analyse producteur-consommateur comparable à celle de tous les autres échanges » (ibid., p. 110). Remarquons que cette expression renforce plutôt qu'elle ne contredit le parallèle entre écriture et action. Le cours développera l'idée de la littérature comme forme d' « anti-action ». De même, aux feuillets [81] $v^{\circ}$-[90], la possibilité d'envisager une équivalence entre littérature et action va expressément émerger.

25. « [...] on comprend les / autres et on les fait être. Mais le propre de cette parole est / d'être parole indirecte, le lyrisme n'y est jamais enfermé, te / silence et la solitude n'y sont rompus que par la fulguration / du bonheur, qui est silence ou œuvre, de toute façon n'est pas / vie » (ibid., p. 214).

26. Ibid., p. 236.

27. Ibid., p. 131. 


\section{6 niziz: st} wirie. lawe i hatulin
XJV ह 5

130

\section{tice Eaire th viru}

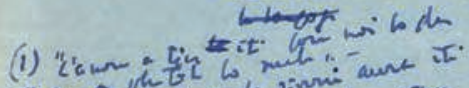

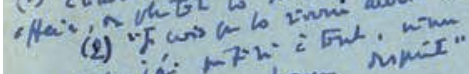
of ia tar hom A caner wom hom

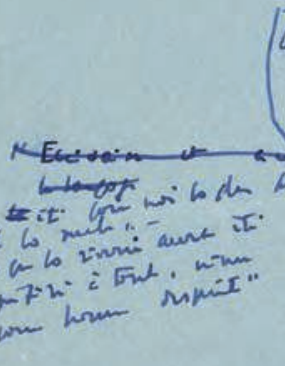

oriectinis

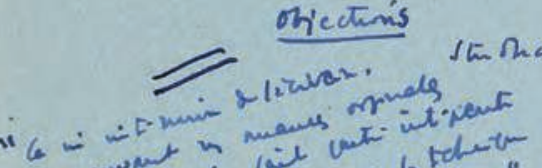

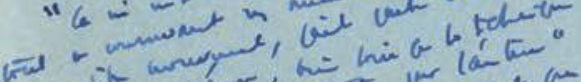

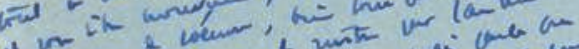

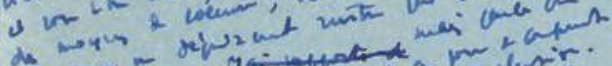

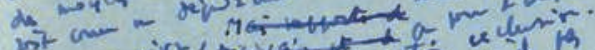

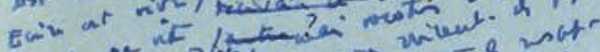

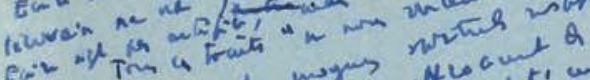

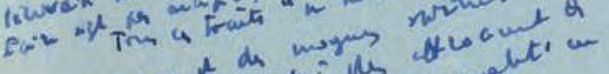

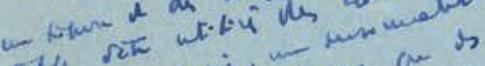

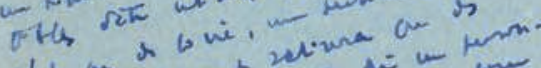

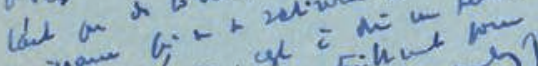

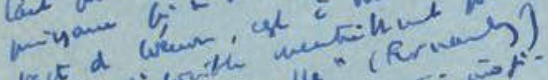

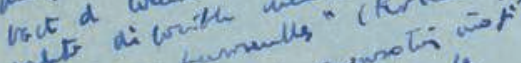

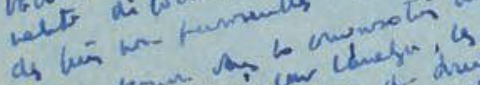

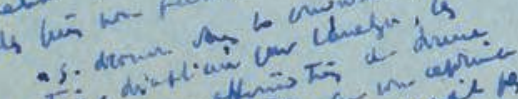

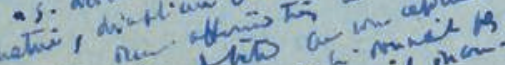

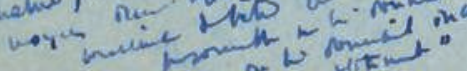

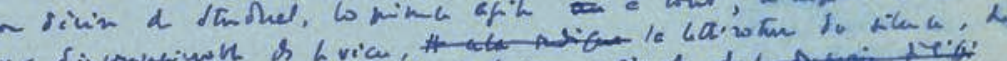

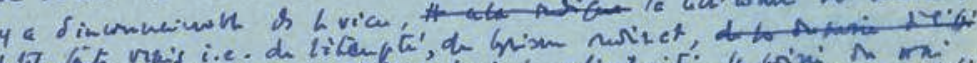

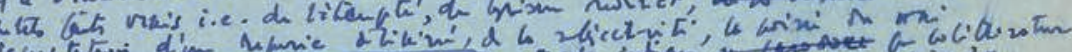

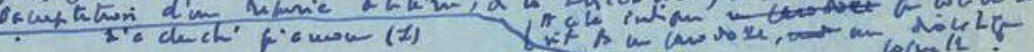

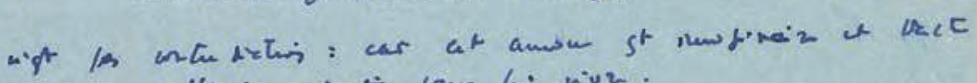
divin ut ti for liven.

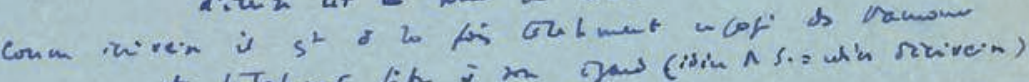

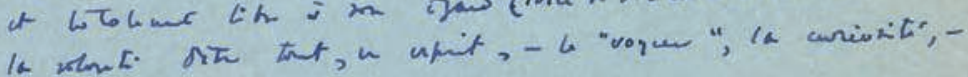
liven wes ame 2 imb'er.

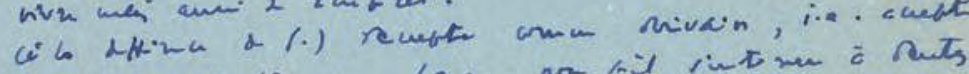

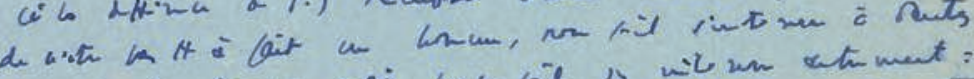

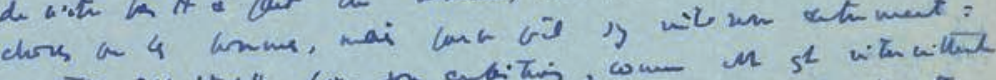

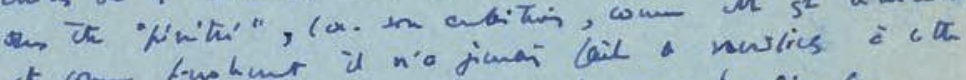

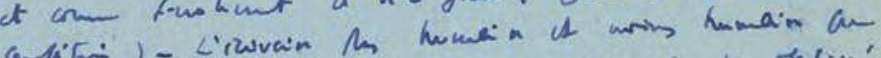

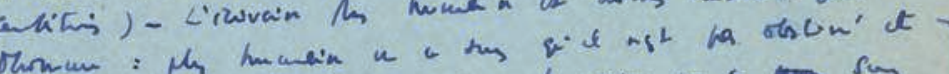

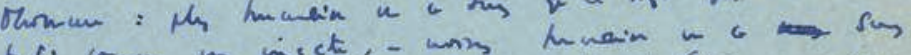

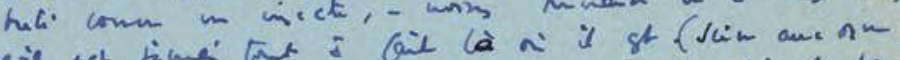

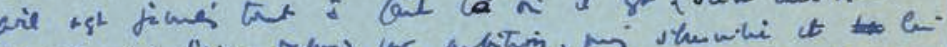

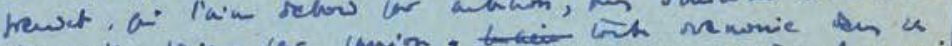

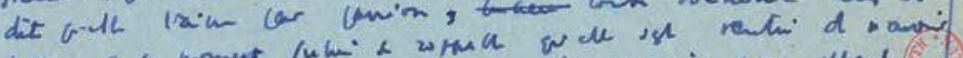

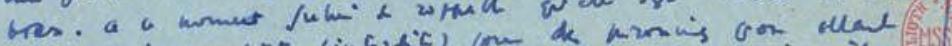

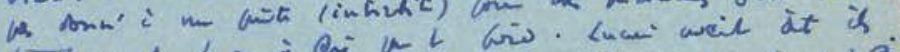

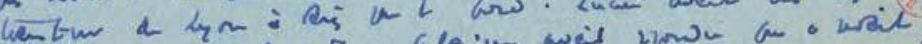

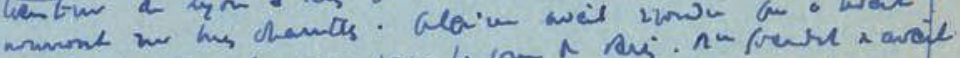

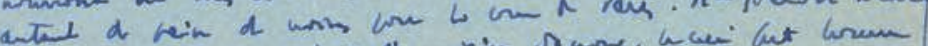

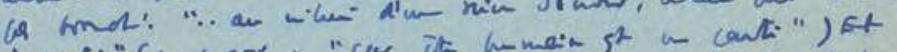

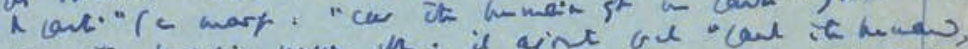

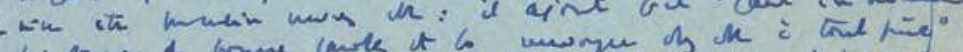

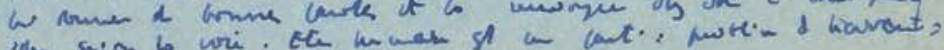

Fig. 9 : Maurice Merleau-Ponty, notes pour le cours « Recherches sur l'usage littéraire du langage » (BnF, fonds Merleau-Ponty, NAF 26994 MF 9846, fo 130 ro) 
de la littérature par une autre vérité à laquelle on l'incorpore-

rait. [...] Ce que c'est que dire ou écrire en littérature] (fig. 10) 28 .

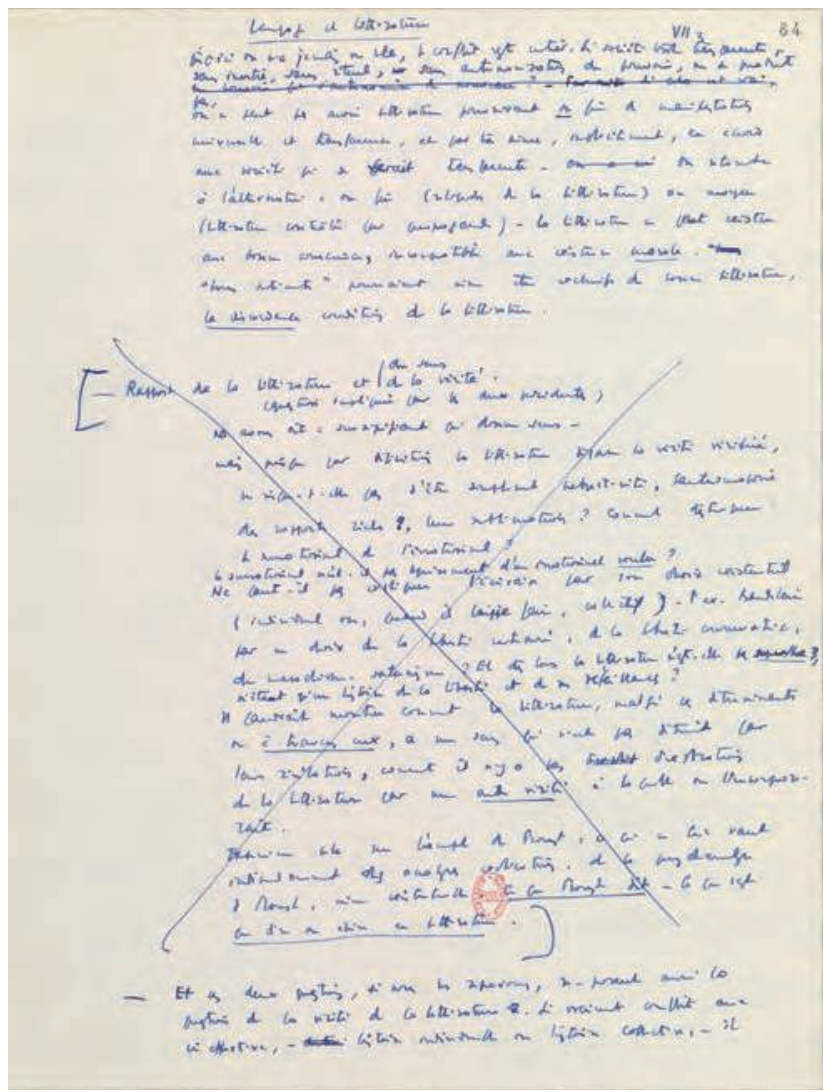

Fig. 10 : Maurice Merleau-Ponty, notes pour le cours « Recherches sur l'usage littéraire du langage »

(BnF, fonds Merleau-Ponty, NAF 26994 MF 9846, fo 84 ro)

\section{Michel Foucault : quelle vérité pour (l' enseignement de) la philosophie?}

À la lumière de la configuration autoréflexive que nous avons essayé de mettre en évidence, le cadre institutionnel et symbolique de l'enseignement au Collège de France semble rendre difficile le positionnement épistémologique de l'orateur par rapport à sa propre œuvre, cette dernière étant en même temps le lieu et l'objet de l'exposé, comme l'exemple de Valéry nous a permis de le voir. Aussi, comme le cas de Merleau-Ponty le laissait comprendre, une telle prise de parole implique un questionnement sur les limites de la discipline investie par l'enseignement, telle qu'elle se donne historiquement au moment de la profération du cours.

Nous demandons souvent au savoir plus que nous ne sommes prêts à lui reconnaître en termes de cohérence et de fondement épistémologique. Nous exigeons des institutions qui incarnent la fiabilité d'un savoir collectif de produire un discours théorétiquement fondé, au moment même où celui-ci se voit confié non pas à la transmission écrite, mais à un enseignement oral, qui se nourrit nécessairement de la contingence de la situation et de l'horizon d'attente d'un public culturellement et sociologiquement déterminé. Dans une telle configuration, la possibilité d'un discours pleinement véridique semble bien délicate, et cela notamment en philosophie, où l'on a fait depuis longtemps le deuil d'un savoir systématique et capable même de structurer tous les autres.

Pour conclure ces quelques considérations, nous nous attarderons sur un cas particulièrement emblématique. Il s'agit du cours prononcé par Michel Foucault au Collège de France en 1984, significativement intitulé «Le courage de la vérité 29 ». Une fois de plus, nous sautons d'une génération à la suivante, mais cette fois nous passons de leçons préliminaires au dernier cours que Foucault sera à même de prononcer au Collège. Si Foucault aborde avec le plus grand humour les questions relatives à sa santé, s'amusant à faire rire son auditoire dès l'ouverture de la première séance, il se fait très sérieux quand il en vient à l'éclairer sur les formes imposées à son enseignement au sein du Collège de France. Notons d'ailleurs que

\section{Ibid., p. 153.}

29. Nous choisissons pour ce faire d'interroger les archives sonores disponibles en ligne : <http://michel-foucault-archives.org/?Cours-aucollege-de-France-1984-Le>. Ce cours est également disponible dans l'édition procurée par Frédéric Gros : Michel Foucault, Le Courage de la vérité. Le gouvernement de soi et des autres II, Paris, Gallimard/Éditions du Seuil, coll. « Hautes Études », 2009. Quant aux manuscrits, ils sont consultables depuis 2013 au département des Manuscrits de la Bibliothèque nationale de France. Voir ici même, p. 52 et 53, la reproduction de deux feuillets issus de ce fonds. 
tout au long de ces cours on entend Foucault dialoguer directement ou indirectement avec son public, se souciant des conditions matérielles de l'écoute dans une salle bondée, non moins que des enjeux pédagogiques impliqués par la transmission d'un savoir qui touche aux structures et aux formes de notre civilisation.

Dans la perspective qui est la nôtre, il n'est pas anodin qu'en ouverture du cours, dans la leçon du 1er février 1984, Foucault rappelle que, par statut, les cours au Collège de France «sont et doivent être [...] des cours publics » et que «n'importe qui, citoyen français ou non, d'ailleurs, a le droit de venir [les] écouter ». L'orateur précise également que «les professeurs ont l'obligation de faire part régulièrement dans leurs cours publics des recherches qu'ils font ». Or, d'après Foucault, cela pose un problème, puisque l'exposé de certains sujets (et notamment de ceux sur lesquels il est en train de travailler : la possibilité, le danger, la responsabilité d'un discours vrai tenu au sein d'une collectivité politique et morale, virtuellement adressé à tout « citoyen ») est une chose délicate, dont il préférerait traiter au sein d'un séminaire restreint à un groupe limité d'étudiants et de chercheurs qui pourraient alors travailler collectivement. À plusieurs reprises, nous entendons ainsi Foucault, qui entre-temps devra renoncer au séminaire «ésotérique » qu'il tenait parallèlement à son enseignement magistral « exotérique », se plaindre de ne pas pouvoir se consacrer à cette activité qu'il juge fondamentale.

Foucault a expérimenté au cours de sa carrière à quel point son enseignement et sa parole pouvaient déranger l'ordre social existant. Il a dès lors pleinement conscience d'une tension entre la situation pédagogique dans laquelle il se trouve et les contenus possibles de son enseignement. Cela est d'autant plus important pour nous que le cours tourne autour de la question de la paresia que Foucault avait commencé à aborder l'année précédente. Si Foucault n'est pas Socrate, si la situation d'enseignement n'implique pas de risque direct pour sa vie, ce qui est ailleurs défini dans ces mêmes leçons comme une condition nécessaire du dire vrai tel qu'il avait été conçu dans le monde grec $^{30}$, le philosophe se pose incontestablement la question de la forme et des limites de la véridicité de son propre enseignement. Si Socrate, dans la lecture de Foucault, choisit d'abord de ne pas prendre part à l'assemblée pour ne pas risquer d'être condamné à mort et pouvoir exercer auprès des citoyens interrogés, un par un, un véritable exercice de vérité, il assume une responsabilité évidente face à la collectivité, responsabilité liée à l'implication de l'autre dans la pratique de la véridiction.

D'ailleurs, la forme même de la transmission pédagogique est prise en compte comme l'une des quatre modalités du dire vrai attestées dans l'histoire de notre civilisation, qui s'avèrent donc non moins présentes dans les pratiques de notre culture contemporaine que dans la société grecque dans laquelle elles avaient trouvé leur origine $^{31}$. L'enseignement académique est selon Foucault la modalité « technique » du dire vrai ; il se spécifie dans un rapport direct à la transmission. $\mathrm{L}^{\prime}$ « exercice » requiert à la fois une techné et son partage avec un ou plusieurs disciples. Les sujets qui se trouvent à pratiquer une telle forme de véridiction « [...] détiennent ce savoir et le professent, c'est-à-dire, ils sont capables de l'enseigner aux autres ». Mieux, ils sont tenus à partager ce qu'ils savent, puisqu'ils ont eux-mêmes reçu d'un maître la technique dont ils disposent. Dans l'analyse de Foucault, le technicien, au moins dans sa forme grecque, possède un devoir de «parole » : il est « tenu à dire ».

30. Voir la leçon du 1er février, première et deuxième heures, et la leçon du 15 février, première et deuxième heures.

31. Voir la leçon du 1er février, deuxième heure. Remarquons qu'encore une fois, aussi bien en clôture de la première heure du cours, juste après avoir énoncé les quatre modalités du dire vrai (celle du sage, du prophète, du paresiaste et du « professeur, ou du technicien, le dire vrai de l'enseignement »), qu'en ouverture de la deuxième heure, Foucault fait référence aux conditions matérielles de son enseignement, et notamment la présence d'un trop vaste public pour permettre le confort de l'auditoire, aussi bien qu'à la «paresia toujours aveugle des institutions », ce qui ne manque pas de faire rire ses étudiants. Sans pouvoir trop entrer dans les détails de cette dimension autoréflexive du cours de Foucault, remarquons que, malgré le fait que la véridiction paresiastique est sans doute plus proche de ce que le philosophe semble vouloir faire ou qu'il propose du moins comme modèle de travail et de compréhension par l'échange, l'identification à ce savoir technique est sans doute attestée, encore une fois par une plaisanterie, au début de la deuxième heure du cours du 1er février. En dressant la liste des personnages qui, dans les dialogues socratiques de Platon, incarnent cette figure, Foucault en donne quelques caractéristiques et, en toussant, il ajoute « et qui tousse ». D'ailleurs, Foucault souligne le fait que, historiquement, les quatre formes de véridiction indiquées se donnent aussi dans leur mélange. 
Le dire vrai, dans l'enseignement comme dans la véridiction proprement paresiastique, ne peut donc advenir que face à autrui, dans la transmission d'une parole qui trouve ce qu'elle trouve en raison des conditions dans lesquelles elle se trouve. Dans l'analyse de Foucault, cet autre sujet est à la fois le lieu, le moyen et l'objet du geste socratique qui ouvre, non sans risques, une vérité possible. Cet autre, en position d'apprentissage, est donc aussi la condition nécessaire de l'exercice de la paresia, comprise comme pratique dont la performativité s'exprime une fois de plus dans l'oralité, et dont le dialogue, en tant que forme littéraire écrite, n'est que la traduction. Or, c'est justement cette performativité qui permettait à Platon, dans l'Alcibiade, de délimiter le territoire d'une métaphysique à venir, comprise comme un face-à-face de l'homme et de son âme, de son essence et de sa nature. C'est elle aussi, comme l'atteste le Lachès, qui conduira au souci de soi et plus particulièrement au soin esthétique de sa propre vie, à l'attention portée à la forme et au style de sa propre existence. Par là, le discours de Socrate, et peut-être de tous ceux qui exercent cette parole qui tente, dans la relation à autrui, d'atteindre une vérité, révèle une duplicité qui loin d'être hasardeuse, incarne, selon Foucault, une ambiguïté cruciale pour l'« histoire de la philosophie occidentale 32 ». Or, si dans ces leçons Foucault est conscient de découvrir un clivage crucial de la philosophie occidentale, il ne peut qu'être conscient aussi de l'importance de prononcer un cours dans lequel ce même clivage est analysé.

La transmission semble alors ressembler à ce jeu d'enfants où un message passe de bouche à oreille, jusqu'au moment où il arrive à destination, déformé dans son sens et dans sa forme. C'est toutefois à l'oralité d'un cours, et même d'un cours ouvert à tous, que Foucault confie sa découverte, ainsi que son point de vue sur cette tradition qui le précède et l'appelle à prendre la parole. Et c'est à Socrate, auteur sans livres, que Foucault, lecteur et auteur, fait appel pour comprendre ce qui, dans ce message qui depuis des siècles nous a été transmis, semble encore et toujours appeler une interprétation nouvelle. Comme si la vérité - si encore elle peut être - ne pouvait que se dire, et non pas (plus?) s'écrire.

32. Cours du 29 février 1984, première heure. 